\title{
Hypothèses sur une énonciation photographique en langue
}

Hypotheses about a photographic enunciation in the language

\section{Joséphine Vodoz}

Université de Lausanne - Unil - Lausanne - Suisse

\begin{abstract}
Résumé: Á partir d'une étude sur le dispositif énonciatif de la photographie, nous ferons ici l'hypothèse que certaines formes langagières fonctionnent de façon similaire au procédé technique. On s'intéressera en particulier aux formes que le locuteur utilise pour témoigner et sur la description qu'en donnent la Théorie des blocs sémantiques et la Théorie Argumentative de la Polyphonie.
\end{abstract}

Mots-clés : énonciation ; photographie ; témoignage ; Théorie des blocs sémantiques, Théorie argumentative de la polyphonie.

Abstract: In this paper, I aim to demonstrate that some language forms, in particular forms that are used to testify, show a similar construction as the photographic enunciation. After presenting important notions in order to understand the photographic enunciation device, I focus on what could be their language equivalent. To conclude, I applicate theses hypotheses through a brief analysis of the poem "Un Rêve" by Aloysius Bertrand.

Keywords: enunciation, photography, testimony, Théorie des blocs sémantiques, Théorie argumentative de la polyphonie. 
A partir d'une étude sur le dispositif énonciatif de la photographie, nous ferons ici l'hypothèse que certaines formes langagières fonctionnent de façon similaire au procédé technique. On s'intéressera en particulier aux formes que le locuteur utilise pour témoigner et sur la description qu'en donnent la Théorie des blocs sémantiques et la Théorie Argumentative de la Polyphonie.

L'énonciation, en tant que marque de la subjectivité du locuteur dans son énoncé, a été peu examinée dans les études photographiques jusqu'ici. II faut d'ailleurs la dissocier immédiatement de la question du style, qui elle s'est maintenant épanouie dans les ouvrages d'histoire de l'art et de la photographie ${ }^{1}$. Comme en langue, les marques de la subjectivité ne se réduisent pas à l'expression d'une «personnalité », elles sont bien en amont et seront notre seul intérêt ici.

Peut-on retrouver des marques de subjectivité dans une image photographique ? II existe beaucoup moins d'écrits sur ce sujet. La légitimité même de la question n'est pas une évidence. Elle apparaît pour la première fois pendant l'effusion théorique qui naît autour de la photographie dans les années 1960-1970, et elle relève bien plus de sa possibilité même que de ses modalités. En effet, la photographie souffre encore à l'époque d'un préconçu né en même temps qu'elle, alléguant qu'elle ne serait qu'une pure reproduction du réel. André Bazin le formule synthétiquement dans son célèbre essai sur le médium : « tous les arts sont fondés sur la présence de l'homme; dans la seule photographie, nous jouissons de son absence » (1958, p.15). Dans ce contexte, l'énoncé photographique est envisagé comme intégralement dénotatif : "En somme, de toutes les structures d'information, la photographie serait la seule à être exclusivement constituée et occupée par un message "dénoté" » (1961, p.129).

\footnotetext{
1 Van Lier voyait déjà dans ce qu'il appelle des « effets de champs» (de texture) des moyens de reconnaître le photographe à l'origine du cliché (Henri Van Lier, 1983 :23). D'après Jean-Claude Lemagny, avec quelques réglages techniques, on pourrait « faire des Nadar, faire des CartierBresson » (1992:52). II nous semble que ceci est admis dans les monographies d'histoire de l'art. Au même titre qu'un peintre, un photographe peut se reconnaître par la récurrence de certains traits formels, graphiques.
}

Dans le but de revenir sur ce présupposé et de définir strictement ce que pourrait être une " énonciation photographique " en langue, nous reviendrons sur certaines notions propres à la théorie photographique. De ces notions pourront être extraits et réduits des paramètres auxquels nous trouverons des échos, voire des équivalents pertinents dans certains faits de langue. Nous verrons également que ces faits de langues ont assez en commun pour que l'on puisse proposer un modèle énonciatif langagier qualifiable de photographique et nous l'appliquerons sur un poème en prose d'Aloysius Bertrand, “Un Rêve » (1842).

\section{Indice et index}

Pour commencer, il est nécessaire de s'arrêter sur la notion d'indice. En effet, il apparaît qu'une étude de la subjectivité dans l'image photographique ne peut se faire qu'après une définition, et donc une délimitation, de la part indicielle (ou dénotative) de la photographie. On s'accorde à définir l'indice, d'après Pierce (même s'il est rarement cité), comme un signe renvoyant à un objet qui l'a physiquement affecté. Dans le cas de la photo, c'est le contact entre les rayons lumineux de l'objet photographié avec la pellicule qui la définirait comme un indice. C'est cette caractéristique de la technique photographique qui a pu pousser la critique à la définir comme une pure « référence » au réel qu'elle a capturé et, par extension, comme une attestation de cette réalité.

II faut effectivement, pour qu'il y ait une photographie, que quelque chose soit photographié, c'est-à-dire, dans l'état actuel de la technique du moins - et cela semble peu susceptible de changer, ou alors on ne parlera plus de «photographie »-, qu'il y ait eu contigüité physique entre l'objet de la représentation et l'appareil. S'il y a eu contigüité physique avec cet objet, cet objet existe. Nous retiendrons ici du terme « indice » moins ce qu'il décrit en termes de rayonnement lumineux ou de phénomènes optiques qu'une idée du raisonnement qui fait dire d'une chose qu'elle est un indice au sens commun du terme : s'il y a une photo de $x$, c'est que quelqu'un l'a prise ; c'est que quelqu'un (ou l'appareil) 
a côtoyé $x$; c'est que $x$ a existé. Cette définition nous semble applicable à la photographie en général (argentique ou numérique), en excluant évidemment tout élément relatif à la retouche (manuelle ou numérique). On revient quelque part à la définition de Barthes (synthétisée dans l'expression «ça a été ») qui touchait déjà à ce noyau logique ${ }^{2}$.

Du reste, la photographie n'est pas qu'une pure attestation de ce qui est. Le français, contrairement à l'anglais, fait la différence entre indice et index; Van Lier applique le premier cette distinction à l'image photographique. II y aurait, dans l'image, une part d'indiciel (une inscription non intentionnelle de la nature sur la pellicule) et une part d'indexical, modelée par des intentions conscientes du photographe. Il définit cette dernière ainsi:

Des index peuvent indiquer certaines portions privilégiées des empreintes, et donc aussi des indices photographiques en les accentuant ou en les orientant. [...] C'est, par exemple, les noircissements ou les éclaircissements de certaines portions de l'empreinte lors du développement. Des choix de pellicules ou d'impression, ou une diaphragmation, qui montrent qu'on a voulu attirer l'attention sur la lumière du matin ou du soir, ou sur la qualité d'ombre du sous-bois. (1983, p.23)

Il s'agit en fait de tout ce qui, dans l'image, figure les choix opérés par le photographe pour en diriger la prise. Selon le philosophe, une image pourrait en posséder comme elle peut en être dénuée (auquel cas la photo est purement indicielle). Ainsi, l'intentionnalité qui crée les index en fait des signes au sens fort du terme, contrairement aux indices qui se caractérisent par leur passivité. II ajoute encore une remarque concernant la spécificité de l'articulation de ces deux composants de l'image:

Ce qu'il y a de remarquable dans la photo c'est que, quand elle comporte des indices et des index, ceux-ci ont entre eux une relation extrêmement intime. [...] les vrais index photographiques, comme le cadrage, l'éclaircissement, l'obscurcissement, la situation dans le champ etc., signalent les indices du dedans, dans leur texture et leur

\footnotetext{
2 J'appelle « référent photographique », non pas la chose facultativement réelle à quoi renvoie une image ou un signe, mais la chose nécessairement réelle qui a été placée devant l'objectif, faute de quoi il n'y aurait pas de photographie. La peinture, elle, peut feindre la réalité sans l'avoir vue. [...] Et puisque cette contrainte n'existe que pour elle, on doit la tenir, par réduction, pour l'essence même, le noème de la photographie. (1980:120)
}

structure, qu'ils accentuent et orientent. (Ibid., 24)

II est ainsi possible de distinguer, conceptuellement, deux plans de l'énoncé photographique, correspondant à deux facettes (ou deux traductions possibles) du signe piercien index (en anglais). Cependant, il est impossible, concrètement cette fois, de distinguer ces deux plans dans une image. Les deux éléments, sur le papier, ne font qu'un. Si je prends une photographie d'un visage en effectuant la mise au point sur l'arrière-plan, le flou du visage est tout en même temps un flou (une énonciation) et un visage (un contenu). Les choix optico-chimiques qu'offre la photographie affectant obligatoirement la totalité de l'image, ils ne sont pas isolables, dans l'objet qui en résulte, sous forme de signes discrets.

Plus récemment, Laurent Jenny reprend cette (in)distinction dans un article où est posée explicitement la question d'une possible énonciation photographique:

La photographie est indissociable de l'image construite qu'elle constitue et cette construction, aussi sommaire soit-elle, est la marque d'un sujet photographe qui organise la deixis. L'œil est donc guidé dans un champ déictique différencié qui renvoie cette fois non plus à l'empreinte de la nature mais à l'attention du sujet photographe. Seul ce champ déictique différencié relève de quelque chose qu'on pourrait appeler énonciation. Mais on voit aussi comment les deux plans déictiques, indiciel et indexical, adhèrent l'un à l'autre, sont donnés simultanément et apparaissent indissociables. (2016, p.262)

Jenny caractérise ces deux sortes de signes (indices et index) en leur attribuant un référent propre. L'indiciel serait ce qui renverrait à l'empreinte de la nature, alors que l'indexical serait signe de l'attention du photographe. On retrouve ainsi une sorte de reformulation du couple traditionnel contenu/énonciation, mais dont la particularité réside dans leur indissociabilité : d'après Jenny, c'est ce qui distinguerait la photo d'un système comme celui du langage ${ }^{3}$.

\footnotetext{
3 «Dans l'ordre de la photographie, l'équivalent des subjectivèmes, c'est l'ensemble des choix optico-chimiques que peut déterminer le photographe. [...] À la différence des subjectivèmes linguistiques, il faut remarquer que ces choix
} 
L'image indicielle a aussi été envisagée comme un déictique. Or, elle ne l'est qu'au sens lâche du terme: sa référence est fixe et ne change pas selon les contextes. Une fois prise, ce n'est plus l'icimaintenant ni du photographe, ni de quiconque aura le cliché devant les yeux. Fondamentalement, l'image photographique fait toujours « signe » de la situation d'énonciation qu'elle a capturée ; ou plutôt, elle ne peut logiquement que représenter sa propre situation d'énonciation, elle-même logiquement comprise comme s'étant déroulée dans le passé. On préfèrera ainsi au terme " déictique " le constat qu'une photographie évoque toujours sa propre situation d'énonciation, et, par extension, son dire.

II faut ensuite noter que ce dire est toujours une actualité. En effet, théoriquement, l'image ne fait en réalité aucunement signe d'un moment passé, à part par le simple fait qu'elle existe, et donc qu'elle a été prise. L'usage du passé composé dans le formule de Barthes ("ça a été ») nous paraît ainsi trompeur, car sa qualité de temps du passé ne trouve pas d'équivalent théorique dans l'image photographique. Ce rapport nostalgique à l'image est en fait plus un fait de réception que d'ontologie. Une photographie représente ainsi moins un «moment passé » qu'une sorte de «moment qui fut présent », le passé simple n'étant suggéré que par le fait que la photographie est matériellement présente devant nous. On fera plus tard un rapprochement entre ce phénomène et son seul équivalent linguistique, le présent d'énonciation sous forme écrite, enregistrée ou rapportée.

\section{Les formes témoignantes}

Afin de faire le pont entre ces remarques sur la photographie et la langue, on se penchera sur les diverses formes qu'un locuteur peut utiliser pour témoigner. En effet, la description qu'en font la TBS et la TAP nous semblent offrir une possibilité de mise en écho avec ce que nous avons dégagé à propos de la photo.

Nous incluons dans l'appellation «formes témoignantes» le verbe témoigner, dans sa

version

affectent la totalité de l'image photographique (le choix du cadrage définit aussi les limites de la visibilité ", ibid., 265. performative (témoigner que) et prédicative (témoigner de). Mais aussi, en suivant la TBS, tous les énoncés qui évoquent la signification de rapporter, au sens de faire un rapport $d e^{4}$ ainsi que certaines phrases exclamatives. La TBS va nous permettre à la fois de décrire précisément ce que ces faits de langues ont en commun, en quoi ils relèvent d'un acte de témoignage et en quoi cela les rend «photographiques ».

Le verbe témoigner, et les énoncés évoquant faire un rapport de, ont pour propriété de concrétiser un aspect argumentatif du type : X DONC DIRE « $X »$. En effet, les énoncés qui contiennent l'un ou plusieurs de ces éléments auront tous pour effet de signifier, au moins en partie: "ce que je communique est la cause même de mon énonciation » et ainsi de présenter leur énonciation comme étant provoquée par l'existence des faits racontés ${ }^{5}$. La phrase exclamative, elle, peut reposer sur le même principe. On dit d'une exclamation qu'elle se retient ou s'étouffe, en bref, elle se contrôle difficilement: la phrase exclamative, elle, se présente comme une énonciation forcée par ce qui la cause. Quand je dis «Qu'il fait chaud!", je prétends que la chaleur ambiante est la seule raison pour laquelle je parle (alors que je cherche peut-être à combler un blanc dans la conversion). Ainsi, l'énoncé «Qu'il fait chaud! " évoque il fait chaud donc je dis qu'il fait chaud, et concrétise X DC DIRE «X $\mathrm{X}$.

Pour résumer, les énoncés qui disent témoigner ou faire un rapport de, ainsi que les phrases exclamatives concrétisent l'aspect $x$ DONC DIRE « $X »$. Souvent, en particulier dans les énoncés qui « rapportent», le dire est accompagné d'une perception : on dit dire quelque chose parce que ce quelque chose a été vu, entendu, perçu. Ainsi, je peux communiquer «Qu'il fait chaud! » en disant « J'ai si chaud!»; dans le deuxième cas, le fait que la

\footnotetext{
${ }^{4}$ Les énoncés dont on peut faire une paraphrase argumentative avec faire un rapport de, que la TAP regroupe sous l'appellation «ton de reportage ».

5 «Raconter à son conjoint ses difficultés à trouver un emploi n'est pas témoigner, même si le propos du récit est caractéristique d'une époque : le locuteur ne déclare pas dire à cause de la difficulté à trouver un emploi. II en va différemment pour le journaliste reporter, qui prétend que l'évidence des faits le pousse à dire et communique ainsi [je l'ai vu donc je le dis] », Dinah Ribard, Marion Carel (2016), 44.
} 
chaleur est un ressenti subjectif est accentué par le verbe « avoir » (qui dans « avoir chaud» tient lieu de perception). Ainsi, lorsque le locuteur témoigne d'un évènement, sa propre perception de l'évènement peut apparaître dans l'énonciation, ou non. Les énoncés peuvent donc concrétiser $X$ DONC DIRE « $X$ » ou PERCEVOIR $X$ DONC DIRE « $X »$. Malgré la variation, on reconnaît la similitude, la répétition $d u \quad x$, à la fois cause et signifié de l'énonciation.

Dans cet aspect type des formes témoignantes, on voit que les deux « $X »$, par effet d'homologie, acquièrent le statut d'«indice » du réel. En effet, en présentant son énonciation comme une la pure répétition d'un fait où d'un évènement perçu, le contenu de l'énoncé, le locuteur certifie ne dire que la réalité des choses mêmes, ne dire les choses que parce qu'elles sont et donc comme elles sont. Sous cet angle de vue, l'analogie avec le plan indiciel de la représentation photographique paraît faire tout à fait sens, d'autant plus si l'on retient la définition minimale que l'on a donnée de l'indicialité. En tant que déduction logique (si $x$ a été photographié, alors $x$ existe), l'indicialité pourrait presque se formuler en terme argumentatif : x photographié DC x existant, ce qui reprend presque, en miroir, l'aspect des formes témoignantes ( $\mathrm{X}$ a existé $\mathrm{DC}$ « $\mathrm{X}$ » est dit).

Par ailleurs, les formes qui nous intéresseront seront celles qui sont investies. En suivant la TAP, on ne considérera en effet que les énoncés apparaissant sur le mode du conçu, c'est-à-dire directement assumés par un locuteur (« je ») individuel.

Dans les formes témoignantes investies, comme l'incarne parfaitement le performatif « Je témoigne que », le « réel », est transmis par le biais d'une médiation du locuteur et de son dire, au contraire des énonciations de type "historique ", où les évènements «se racontent eux-mêmes » pour reprendre les termes de Benveniste (1966, p. 241). Cependant, le dire ne fonctionne ici que comme simple support, et non comme une subjectivation du contenu au sens axiologique ou affectif du terme, puisque l'aspect argumentatif des formes témoignantes présente le contenu comme étant calqué sur les évènements qu'il relate.

\section{Le présent d'énonciation}

On ajoutera encore quelques remarques sur ces formes investies (ou, en d'autres mots, qui trahissent une énonciation) à partir de la description du présent d'énonciation que fait Guy Serbat dans deux articles publiés dans I'Information grammaticale (1980, p. 1988).

Le linguiste revient, dans un premier temps, sur une description commune du présent grammatical comme forme atemporelle, dont la valeur s'adapte aux informations contextuelles données par l'énoncé ou les énoncés voisins. Elle serait, selon l'auteur, invalide. En effet, nous utilisons le présent pour signifier «l'actualité », et ce, même sans complément: « II fait chaud», sans autre précision, sera compris comme "II fait chaud maintenant». C'est ici que la thèse de Serbat gagne en intérêt pour nous : au lieu de conclure, comme on le ferait peutêtre intuitivement, que le présent est fondamentalement le temps de l'actualité (d'où son nom) mais qu'il peut tout de même changer de valeur selon les contextes, Serbat propose de considérer cet « effet d'actualité » comme une déduction logique, forcée par la nature de l'acte énonciatif lui-même. II rappelle dans ce but une vérité simple qui est que, derrière tout énoncé, il y a un locuteur. De fait :

Ce locuteur est forcément installé dans un nunc (« maintenant») dans un hic (« ici »); ce sont là deux conditions existentielles dont nul ne peut s'évader, et qui restent constamment implicites. C'est pourquoi, si l'énoncé expose des faits étrangers à l'ici et au maintenant, le locuteur est obligé de le spécifier expressément, sous peine d'induire en erreur. [...] Mais lorsque rien n'invite à abandonner ce cadre, tout énoncé est obligatoirement rapporté aux conditions naturelles et nécessaires de sa production. (1980, p. 38)

Ainsi, dans l'énoncé « II fait chaud », ce n'est pas le présent du verbe qui signifie le présent. La lecture que nous ferons de cet énoncé comme un présent n'est pas déclenchée par un signe spécifique: ni par aucun complément ni par la désinence verbale (sinon, comme le fait remarquer Serbat, une désinence du présent dans un énoncé au futur serait contradictoire). Cette lecture est provoquée par un phénomène d'ordre psycho- 
philosophique plus que grammatical, il ne se laisse pas « isoler » dans une forme précise. On retrouve ici une autre similarité avec le photographique: l'impossibilité pratique de discriminer, dans une représentation, le plan du contenu du plan de l'énonciation : dans « II fait chaud. » autant [fait] que [chaud] que tout autre mot qui aurait pu les accompagner (sauf compléments de temps) évoque une énonciation et l'actualité dans laquelle elle a été produite. Pour citer à nouveau le linguiste : « le NUNC de l'énonceur colore, imprègne, tout l'énoncé » (1988, p. 34).

Comme pour une photographie, un énoncé au présent d'énonciation ne peut, pour être intelligible, qu'être lu comme étant la production d'un «je-icimaintenant ». II faut, pour bien comprendre Serbat, et la photographie, souligner que ce hic et ce nunc n'ont aucunement besoin d'être définis, précis comme peuvent l'être les compléments de verbes. II ne s'agit pas de « dater » un énoncé du moment où le locuteur s'exprime; l'impression d'actualité est une contingence plus qu'une signification. Elle résulte de la constatation, qui sort de la linguistique à proprement dite, que pour l'homme il n'y a de façon d'être au monde qu'actuelle, et que tout acte énonciatif qu'il produit sera ainsi ramené à ce « NUNC existentiel, primordial » (ibid., 35) Ainsi, hic et nunc renvoient moins à des données spatio-temporelles précises qu'à la présence du locuteur, du «je » dont on les déduit.

Dans ses Cahiers (1965, éd. 1986), Ludwig Wittgenstein différencie quant à lui un « je » objectif (qui interviendrait dans les énoncés prédicatifs), d'un « je » subjectif (on dira énoncés « expressifs »). Le « je » objectif est celui qui se désigne lui-même comme étant l'objet de son propos : dans « je suis seul dans cette pièce », le « je » se décrit lui-même comme étant seul dans une pièce. Dans « Je me sens si seul ! » cependant, le but du locuteur n'est pas de se caractériser, mais d'exprimer sa solitude. Avec un « je » est subjectif, le propos tient donc de la performance plus que de l'affirmation : dans ce genre d'énoncés, le locuteur peut se passer d'auto-désignation. Comme le dit Wittgenstein, dire « j'ai mal » équivaut à « pousser un cri de douleur » (1986, p. 127).

Si cette particularité de la langue ne s'applique qu'avec certains verbes, par exemple les verbes de perception (dans leurs emplois modaux), elle n'est pas uniquement d'ordre sémantique, comme semble le penser Wittgenstein, elle est aussi énonciative. Elle découle du fait qu'interprété au présent d'énonciation, et sans indication contraire, un énoncé ne peut se rapporter qu'au « je primordial » que l'on mentionnait (la désignation est ainsi « superflue » car elle est déjà comprise dans le «temps verbal »).

II faut ajouter que cette relation forcée entre une marque claire d'énonciation et un « je » à sa source ne s'opère pas uniquement au présent. On peut constater par exemple que la phrase exclamative investie est attribuable à un « je primordial », même à un temps du passé. C'est, il nous semble, ce qui explique que l'on ait tendance à percevoir, par exemple dans la prose fictionnelle, un discours indirect libre (un effet de point de vue) dans les phrases exclamatives ${ }^{6}$. Par exemple, dans J'ai vu un papillon ! ou « II y avait un papillon ! », malgré les temps du passé, on comprend ces énoncés comme étant une attestation faite maintenant (dans le maintenant du locuteur) d'une perception passée. On constate donc que le « je primordial » est sensible même quand la perception est exprimée au passé, à condition qu'il y ait un dire communiquant la sensation d'actualité propre à l'énonciation.

Les formes témoignantes concrétisent toujours un aspect argumentatif avec DIRE, et donc une actualité contingente. C'est aussi parce que la perception est par définition perçue par un sujet et est donc intrinsèquement liée à un je percepteur, qui est aussi le locuteur. Dire que quelque chose a été perçu par « je », n'équivaut pas à dire avoir perçu. De la même façon, dire que « je » se sentait seul est une description d'une étape antérieure de soi, alors que dire s'être senti seul est une plainte par rapport à un

\footnotetext{
${ }^{6}$ Chez Flaubert par exemple, dont on sait qu'il travaillait ses textes afin que la présence du narrateur soit invisible, quand une marque d'énonciation comme l'exclamation apparaît on a tendance à l'interpréter comme étant l'énonciation d'un des personnages, et donc un discours indirect libre.
} 
état passé. En d'autres mots, le « je » objectif produit une de dédoublement provoqué par l'attribution (la prédication) : un « je » dit qu'un autre « je » a la caractéristique d'avoir perçu. Au contraire, le « je » subjectif est à la fois celui qui dit, et celui qui a perçu.

En résumé : un énoncé est toujours une trace de son locuteur. Au présent d'énonciation, cette trace conditionne son intelligibilité. Ce que la formulation de Serbat apporte réellement, c'est une présentation du présent d'énonciation comme forme renvoyant aux conditions mêmes de la possibilité d'énoncer. Et c'est ce dernier point qui le lie à la photographie: tout comme une image photographique, un énoncé au présent d'énonciation est une monstration de ses propres conditions logiques d'existence, c'est-à-dire du « je » à son origine : je ne peux les comprendre qu'en les ayant attribuées à un locuteur, où à une situation d'énonciation particularisée.

L'énoncé photographique sera donc celui qui exhibe sa situation d'énonciation, par son dire, et dont l'attribution à un «je » locuteur source est indispensable à son intelligibilité. Même au passé, ces énoncés suggèrent une continuation entre la perception et le dire de cette perception, de par leur subjectivité (au sens de Wittgenstein), de même qu'une photographie demeure toujours un dire (une monstration) actuelle d'une capture passée.

À partir de toutes ces observations, on peut faire un schéma général du type d'énoncé qui réunit des caractéristiques dites «photographiques », et qui donc retiendra notre attention :

\section{JE-LOCUTEUR [(PERCEVOIR) $\mathbf{X}$ DC DIRE « $\mathbf{X} »]$}

Où JE-LOCUTEUR signifie que l'énoncé est investi par une voix de locuteur, et qu'il est donc subjectif au sens de Wittgenstein ;

- Le percevoir est entre parenthèses, car il peut être concrétisé par l'emploi modal de n'importe quel verbe de perception. II peut également se conjuguer au passé (AVOIR PERÇU), si l'énoncé communique une actualité autrement, et peut même disparaître, comme on l'a vu.

Pour reprendre notre analogie avec le photographique, on distinguera les deux $[x]$ qui construisent de l'indiciel, c'est-à-dire qui présentent un contenu comme tautologie pure d'un fait ou d'une expérience vécue, des autres éléments qui en sont le foyer indexical (la monstration du dire). Les énoncés sont donc investis par le locuteur, et apparaissent sur le mode du conçu. Les « [] » qui encadrent l'aspect argumentatif veulent signifier que tout l'extrait participant de l'énonciation photographique se comprend comme énoncé par ce je-locuteur.

\section{L'exemple d'“ Un Rêve ", D'Aloysius Bertrand}

Afin de concrétiser ces remarques, on les appliquera à l'analyse d'un poème d'Aloysius Bertrand, tiré de son recueil Gaspard de la Nuit'.

\section{VII \\ UN RÊVE}

J'ai rêvé tant et plus, mais je n'y entends note. Pantagruel, Livre III

Il était nuit. Ce furent d'abord, - ainsi j'ai vu, ainsi je raconte, - une abbaye aux murailles lézardées par la lune, - une forêt percée de sentiers tortueux, - et le Morimont* grouillant de capes et de chapeaux.

Ce furent ensuite, - ainsi j'ai entendu, ainsi je raconte, - le glas funèbre d'une cellule, - des cris plaintifs et des rires féroces dont frissonnait chaque feuille le long d'une ramée, - et les prières bourdonnantes des pénitents noirs qui accompagnaient un criminel au supplice.

Ce furent enfin, - ainsi s'acheva le rêve, ainsi je raconte, - un moine qui expirait couché dans la cendre des agonisants, - une jeune fille qui se débattait pendue aux branches d'un chêne, - Et moi que le bourreau liait échevelé sur les rayons de la roue.

Dom Augustin, le prieur défunt, aura, en habit de cordelier, les honneurs de la chapelle ardente, et Marguerite, que son amant a tuée, sera ensevelie dans sa blanche robe d'innocence, entre quatre cierges de cire.

Mais moi, la barre du bourreau s'était, au

\footnotetext{
${ }^{7}$ Nous utilisons l'édition de Max Milner, 1980, 145.
} 
premier coup, brisée comme un verre, les torches des pénitents noirs s'étaient éteintes sous des torrents de pluie, la foule s'était écoulée avec les ruisseaux débordés et rapides, - et je poursuivais d'autres songes jusqu'au réveil.

* C'est à Dijon, de temps immémorial, la place aux exécutions

L'« espace-temps » du poème est étrange : trois scènes se déroulent à trois endroits différents (une abbaye, une forêt, le Morimont) et leur juxtaposition suggère qu'elles se déroulent en même temps. Les trois incises mettent, d'autre part, fortement en relation l'évènement raconté et le dire, tout en marquant la différence temporelle. Cette différence qui est d'abord amoindrie par le passé composé des j'ai vu et j'ai entendu (qui peuvent signifier « je suis, maintenant, quelqu'un qui a vu » est ensuite plus affirmée par le passé simple de la troisième strophe: ainsi s'acheva le rêve, ainsi, [maintenant], je raconte. Leur emplacement dans la strophe est également important : placés juste après les «Ce furent », qui introduisent les évènements racontés au passé simple, les incises installent directement une deuxième strate énonciative qui est celle de l'énonciation du locuteur. Ceci a pour effet que les incises « court-circuitent » les «Ce furent» qui, eux, prennent dès lors une fonction d'organisateurs logiques de la représentation plutôt que d'organisateurs temporels d'une succession d'évènements.

II y a donc bien un passé (raconté) et un dire (racontant): il ne s'agit pas, pourtant de rendre l'évènement au présent (de créer un « effet de présence »), mais de rapprocher, voire de superposer fortement les deux strates énonciatives qui se côtoient dans le poème.

Les trois incises, prises ensemble, évoquent l'enchaînement j'ai rêvé de ceci, donc je le raconte et concrétisent l'aspect AVOIR RÊVÉ DC DIRE, paradoxal (c'est-à-dire non prévu par la signification de DIRE). S'il est paradoxal, il peut tout de même être rapproché des aspects que l'on retrouve dans la signification de rapport : le locuteur fait un récit «témoignesque » de son rêve; pas tout un fait un témoignage au sens technique du terme, puisqu'il n'y a en fait que la troisième incise qui concrétise éventuellement un aspect de témoigner, mais cela en reste très proche (partant également du principe que rêver est une forme de perception mentale).

De plus, en reprenant l'aspect: AVOIR RÊVÉ X DC DIRE « $X$ », on s'aperçoit donc que la répétition du même $x$ pose le récit du rêve comme indice du rêve tout en affichant un dire par le je raconte qui tient alors lieu de foyer indexical. En privilégiant une définition argumentative de l'indicialité à une définition sémiotique, on constate que le langage, indépendamment de la véracité ou de la réalité des faits racontés, peut tout à fait simuler cette indicialité. Ici, c'est l'aspect argumentatif des formes témoignantes qui suggèrent cette logique indicielle.

D'autre part, il faut noter que les incises ont un effet sur l'entier du texte. Autrement dit, la situation d'énonciation du locuteur conditionne l'intelligibilité du texte au-delà du seul énoncé où elle apparaît. C'est selon nous ce qui cause la très forte ambiguïté de la dernière phrase du poème : « et je poursuivais vers d'autres songes jusqu'au réveil ».

L'ambiguïté de cette phrase porte sur le «je », puisqu'on ne sait pas s'il est objectif ou subjectif, celui qui est en train de rêver ou celui qui se voit et se raconte en train de rêver (la même ambigüité opère dans la première phrase, // était nuit). On hésite d'une part parce qu'on ne sait à quoi le « et » qui introduit l'énoncé est articulé (soit à ainsi s'acheva le rêve soit à la foule s'était écoulée avec les ruisseaux débordés et rapides, et le tiret y est pour beaucoup dans cette hésitation), mais aussi et peut-être surtout parce que le je locuteur est présent pendant toute la lecture du poème, autant que le je rêvant. Une fois que la situation d'énonciation du je-locuteur a été signalée, par les incises, chaque récurrence de je est susceptible de lui appartenir, car cette situation est en fait comprise comme « situation primordiale ».

Le poème figure donc à sa manière cette indissociabilité photographique entre plan «indiciel » et plan « indexical », c'est-à-dire entre un dit présenté 
comme redondance d'une expérience vécue et une monstration corrélée du dire : elle est figurée dans le poème par l'installation de cette strate énonciative au présent, en arrière-fond d'un propos dont elle conditionne toujours potentiellement la lecture.

$$
* * *
$$

Nous en revenons, pour conclure, à des remarques plus générales.

II ne nous semble pas faux de dire que tout énoncé écrit au présent d'énonciation a quelque chose du photographique, puisque le présent d'énonciation, par définition, où en tout cas dans la perspective de Serbat, partage avec la photographie le fait de n'être intelligible, en tant que tel, uniquement par le biais d'une situation d'énonciation déduite ou actualisée dans l'énoncé. Nous pensons cependant que pour parler $d$ '«énonciation photographique », il faudrait pouvoir réunir deux critères de plus:

Premièrement, il faut qu'il y ait construction d'une indicialité propre aux évènements racontés. Dans le cas des formes témoignantes, cette indicialité est construite par la redondance : en affirmant que le dit est la cause du dire, la description argumentative de l'énonciation crée l'illusion que le dit n'est qu'une répétition, une «photographie » de l'évènement qu'il reprend.

Deuxièmement, il faudrait que la construction de cette énonciation se fasse au niveau textuel (où en tout cas un morceau important de texte). En effet, l'intérêt de ce type de dispositif énonciatif réside également en ce qu'il peut «imprégner», pour reprendre l'expression, une portion plus large de texte que l'énoncé lui-même, impactant sur sa réception et sur son interprétation, comme peuvent en témoigner le poème de Bertrand, voire peut-être d'autres phénomènes comme le discours indirect libre. Dans certains textes, ce type d'énonciation peuvent être interprétées dans un intention poétique spécifique; chez Bertrand par exemple, où les incises participent à un effet d'hypotypose qui s'intègre aisément au projet poétique de son recueil Gaspard de la Nuit.

Ainsi, on espère avoir montré ce qu'un détour par l'image et par la photographie permet de mettre en avant en langue, ici notamment sur la spécificité de l'acte d'énonciation quand il devient condition d'intelligibilité de l'énoncé, ainsi que sur la fonction témoignante de l'énonciation, comprise comme relation argumentative au contenu et avant toute préoccupation de vérité.

\section{Références}

Barthes, R. (1961). Le Message photographique. Communications, $\mathrm{n}^{\circ} 1$, pp.127-138.

Barthes, R. (1980). La Chambre claire : note sur la photographie. Paris : Seuil.

Bazin, A. (1958, éd. 1945). Qu'est-ce que le cinéma ? Ontologie et langage : Paris, Les Éditions du Cerf.

Benveniste, E. (1966). Problèmes de linguistique générale, I. Paris : Gallimard.

Bertrand, A. (1842, éd. 1980). Gaspard de la nuit. Paris: Gallimard, NRF.

Carel, M. (dir.). (2012). Argumentation et polyphonie. De Saint-Augustin à Robbe-Grillet. Paris : L'Harmattan.

Carel, M. (2011). L'Entrelacement argumentatif. Lexique, discours et blocs sémantiques. Paris: Honoré Champion.

Jenny, L. (2016). Y a-t-il une énonciation photographique ?. In Marion Colas-Blaise, Laurent Perrin et Gian Maria Tore (dir.), L'énonciation aujourd'hui. Un concept clé des sciences du langage. Paris : Lambert-Lucas.

Lemagny, J-C. (1992). L'Ombre et le temps : essais sur la photographie comme art. Paris : Nathan.

van Lier, H. (1983). Philosophie de la photographie. Paris : Les Cahiers de la photographie, Hors-série.

Ribard D., Carel M. (2016). Témoigner en poésie. Le cas de Marc de Larréguy. Poétique, n¹79, pp. 3955 .

Serbat, G. (1980). La place du présent de l'indicatif dans le système des temps. L'Information Grammaticale, $\mathrm{n}^{\circ} 7$, pp.36-39

Serbat, G. (1988). Le prétendu "présent" de l'indicatif : une forme non déictique du verbe. L'Information Grammaticale, $\mathrm{n}^{\circ} 38, \mathrm{pp} .32-35$.

Wittengstein, L. (1965, éd. 1986). Le Cahier bleu et le Cahier brun. Études préliminaires aux Investigations philosophiques [1965], traduit de l'anglais par Guy Durand. Paris : Gallimard. 


\section{COMO CITAR ESSE ARTIGO}

VODOZ, Joséphine. Hypothèses sur une énonciation photographique en langue. Signo, Santa Cruz do Sul, v. 44, n. 80, p. 204-213, ago. 2019. ISSN 1982-2014. Disponível em: <https://online.unisc.br/seer/index.php/signo/article/view/14029>. Acesso em:

doi: https://doi.org/10.17058/signo.v44i80.14029. 